\title{
COHOMOLOGY OF LOCAL SHEAVES ON ARRANGEMENT LATTICES
}

\author{
SERGEY YUZVINSKY
}

(Communicated by Jeffry N. Kahn)

\begin{abstract}
We apply cohomology of sheaves to arrangements of hyperplanes. In particular we prove an inequality for the depth of cohomology modules of local sheaves on the intersection lattice of an arrangement. This generalizes a result of Solomon-Terao about the cummulative property of local functors. We also prove a characterization of free arrangements by certain properties of the cohomlogy of a sheaf of derivation modules. This gives a condition on the Möbius function of the intersection lattice of a free arrangement. Using this condition we prove that certain geometric lattices cannot afford free arrangements although their Poincaré polynomials factor.
\end{abstract}

\section{INTRODUCTION}

Let $V$ be an $l$-dimensional vector space over an arbitrary field $K$ and $\mathscr{A}$ an arrangement of $n$ hyperplanes in $V$. For each $H \in \mathscr{A}$ we fix $\alpha_{H} \in V^{*}$ such that $\operatorname{ker}\left(\alpha_{H}\right)=H$. Let $S$ be the symmetric algebra of $V^{*}$ and $\operatorname{Der} S=$ $\operatorname{Der}_{K}(S, S)$ the graded $S$-module of derivations of $S$. The following graded submodule of Der $S$ was introduced by $\mathrm{H}$. Terao [T1, T3] and studied in many papers on arrangements: $D=D(\mathscr{A})=\left\{\theta \in \operatorname{Der}(S) \mid \theta\left(\alpha_{H}\right) \in S \alpha_{H}, H \in \mathscr{A}\right\}$. If the module $D$ is free then the arrangement $\mathscr{A}$ is called free.

One of the most intriguing and challenging problems in the theory of arrangements is to relate $D$ to the intersection lattice $L$ of $\mathscr{A}$. The most general result in this direction is the Solomon-Terao formula [ST] expressing the Poincare polynomial $\pi(L)$ of $L$ through the Poincare series of $D$ and of certain similarly defined graded $S$-modules $D^{p}(p=0,1, \ldots, l)$. To prove this formula the authors observed that the modules $D^{p}$ generate contravariant functors on $L$ having the property of being local (see Definition 1.3). Then they proved that the local property of a contravariant functor $\mathscr{F}$ on $L$ implies that the series

$$
\sum_{X \in L} \mu(X, U) P(\mathscr{F}(X)),
$$

Received by the editors May 29, 1990, and, in revised form, July 25,1990.

1980 Mathematics Subject Classification (1985 Revision). Primary 05B35, 32C40, 13 C15. 
(where $U$ is the maximal element of $L, \mu$ is the Möbius function of $L$, and $P(M)$ is the Poincare series of a graded module $M$ ) has a pole of order at most $\operatorname{dim} U$ at 1 [ST, Proposition 6.10].

In this paper we regard contravariant functors on $L$ as sheaves on the opposite lattice $L^{\mathrm{op}}$ and study the sheaf cohomlogy of the poset $L_{0}=L^{\mathrm{op}} \backslash\{U\}$. First we observe that the series $(*)$ is the alternating sum ("Euler characteristic") of the Poincare series of the reduce cohomology modules $\tilde{H}^{i}\left(L_{0}, \mathscr{F}\right)$. We prove that if $\mathscr{F}$ is local then $\operatorname{dim}_{S} \tilde{H}^{i}\left(L_{0}, \mathscr{F}\right) \leq \operatorname{dim}_{K} U$ which is a generalization of [ST, Proposition 6.10]. Then we restrict our attention to the sheaf $\mathscr{D}$ induced by $D$ and show that $\tilde{H}^{i}\left(L_{0}, \mathscr{D}\right)=0$ for every $i<l-1-\operatorname{pd}_{S}(D)$ (using the symbol $\mathrm{pd}_{S}$ for the projective dimension of an $S$-module). In particular if $\mathscr{A}$ is free then all cohomology groups disappear except maybe the highest one $H^{l-1}\left(L_{0}, \mathscr{D}\right)$. In some cases, for example, for $l=3$, this condition is also sufficient for $\mathscr{A}$ to be free. In any case for a free arrangement the series $(*)$ becomes the Poincare series of a module which implies that its coefficient are nonnegative. Since for a free arrangement the series $(*)$ is defined by $L$ we obtain a new condition on a lattice necessary for this lattice to afford a free arrangement. A previously known condition of the kind was found by Terao [T2] and says that for the lattice $L$ of a free arrangement $\pi(L ; t)=(1+t) \Pi_{i=1}^{l-1}\left(1+b_{i} t\right)$ for certain positive integers $b_{1}, \ldots, b_{l-1}$. Using our condition we are able to prove that many lattices satisfying Terao's condition cannot afford a free arrangement. Some of these results were obtained by Ziegler $[Z]$ by his method of supersolvable resolutions.

\section{Notation}

Here we recall some definitions and set up notation for the rest of the paper. Throughout $\mathscr{A}$ will be an $l$-arrangement of $n$ hyperplanes in a linear space $V$ (of dimension $l$ ) over an arbitrary field $K$. The graded ring $S$ and the module $D=D(\mathscr{A})$ were defined in the introduction. The intersection lattice of $\mathscr{A}$ ordered by reverse inclusion is denoted by $L$. It is a geometric lattice with the rank function given by $\operatorname{rk}(X)=l-\operatorname{dim} X \quad(X \in L)$. The minimal element of $L$ is $V$, its maximal element $\bigcap_{H \in \mathscr{A}} H$ is denoted by $U$. If $\operatorname{dim} U=0$ the arrangement $\mathscr{A}$ is called essential. By $\mu$ we denote the Möbius function of $L$ and by $\pi(L)$ its Poincaré polynomial, i.e., $\pi(L ; t)=\sum_{X \in L}|\mu(V, X)| t^{\mathrm{rk} X}$. Every $X \in L$ defines the subarrangement $\mathscr{A}_{X}$ of $\mathscr{A}$ which consists of the hyperplanes containing $X$. We put $D(X)=D\left(\mathscr{A}_{X}\right)$. We also need the lattice $L^{\mathrm{op}}$ which coincides with $L$ as the set and is provided with the reverse order (i.e., the inclusion) and the poset $L_{0}=L^{\mathrm{op}} \backslash\{U\}$. Not to confuse the orders we use only the symbol $\subset$ for the order on $L^{\text {op }}$.

If $Q$ is an arbitrary finite poset then we view it as a topological space with the topology consisting of all increasing subsets of $Q$, i.e., subsets $R$ such that if $X \in R$ and $Y \geq X$ then $Y \in R$. Then every covariant functor $\mathscr{F}$ from $Q$ to the category of $S$-modules defines a sheaf of $S$-modules on $Q$. We 
denote this sheaf by the same symbol $\mathscr{F}$. If $X \in Q$ then the stalk of $\mathscr{F}$ at $X$ is denoted by $\mathscr{F}(X)$ and $S$-module homomorphism $\rho_{Y, X}: \mathscr{F}(X) \rightarrow \mathscr{F}(Y)$ defined for each pair $X<Y$ from $Q$ is called a structure homomorphism of $\mathscr{F}$. For any prime ideal $I$ of $S$ the localizations at $I$ of the stalks and structure homomorphisms of $\mathscr{F}$ define the sheaf of $S_{I}$-modules which we denote by $\mathscr{F}_{I}$. Similarly any contravariant functor $\mathscr{F}$ defines the sheaf $\mathscr{F}$ on the poset $Q^{\text {op }}$ opposite to $Q$. The crucial example for this paper is given by the sheaf $\mathscr{D}$ on $L^{\mathrm{op}}$ defined by $\mathscr{D}(X)=D(X)$ and the natural embeddings $D(X) \subset D(Y)$ for all $X \subset Y$ from $L$. We keep the symbol $\mathscr{D}$ for the restriction of this sheaf to $L_{0}$.

Let $\mathscr{F}$ be a sheaf of $S$-modules on $Q$. The $i$ th cohomology module of $\mathscr{F}$ is denoted by $H^{i}(Q, \mathscr{F})$. Applying the canonical simplicial resolution from [Go, §6.4] we can view these cohomology modules as the homology modules of the cochain complex $C(Q, \mathscr{F})$ whose $i$-cochains are all functions on the $(i+1)$-tuples $X_{1} \leq X_{2} \leq \cdots \leq X_{i+1}$ from $Q$ with values in $\mathscr{F}\left(X_{i+1}\right)$. The differential of the complex is given by the usual formula

$$
\begin{aligned}
(d f)\left(X_{1}, \ldots, X_{k+1}\right)=\sum_{j=1}^{k}( & -1)^{j-1} f\left(X_{1}, \ldots, \hat{X}_{j}, \ldots, X_{k+1}\right) \\
& +(-1)^{k} \rho_{X_{k+1}, X_{k}} f\left(X_{1}, \ldots, X_{k}\right) .
\end{aligned}
$$

Since the localization at a prime ideal is an exact functor we have $H^{i}(Q, \mathscr{F})_{I}=$ $H^{i}\left(Q, \mathscr{F}_{I}\right)$ for every $i$ and $I \in \operatorname{Spec} S$.

\section{COHOMOLOGY OF LOCAL SHEAVES}

At the beginning of this section $Q$ will be an arbitrary finite poset. First we register two simple facts about the sheaf cohomology on $Q$.

Lemma 1.1. If $Q$ has a unique minimal element, say $m$, then $H^{i}(Q, \mathscr{F})=0$ for every $i>0$ and every sheaf $\mathscr{F}$ on $Q$.

Proof. Indeed the functor of global sections $\Gamma$ is naturally equivalent in this case to the functor $\mathscr{F} \mapsto \mathscr{F}(m)$ which is exact.

In the next lemma we use certain standard notation from sheaf theory (e.g., see [Go, II.1.12 and II.4.16]).

Lemma 1.2. Suppose that $i: R \subset Q$ and $\phi: Q \rightarrow R$ are monotone maps, $\phi i=$ 1 , and $\phi(X) \geq X$ for every $X \in Q$. Suppose also that $\mathscr{F}$ is a sheaf on $Q$ such that $\phi^{*} i^{*} \mathscr{F}=\mathscr{F}$. Then $i^{*}: H^{*}(Q, \mathscr{F}) \rightarrow H^{*}\left(R, i^{*} \mathscr{F}\right)$ is an isomorphism (with the inverse $\phi^{*}$ ).

Proof. Since $i^{*} \phi^{*}=1$ it suffices to prove that $\phi i$ is homotopic to 1 where $\phi$. and $i$ are cochain maps of the complexes $C(Q, \mathscr{F})$ and $C\left(R, i^{*} \mathscr{F}\right)$ corresponding to $\phi$ and $i$ respectively. The most direct way to do this is to exhibit 
a homotopy. For that let us fix $f \in C^{p-1}(Q, \mathscr{F})$ and put

$$
(h f)\left(X_{1}, X_{2}, \ldots, X_{p-1}\right)=\sum_{j=1}^{p-1}(-1)^{j-1} f\left(X_{1}, \ldots, X_{j}, \phi\left(X_{j}\right), \ldots, \phi\left(X_{p-1}\right)\right),
$$

where for every $X \in Q$ we identify $\mathscr{F}(X)$ and $\mathscr{F}(\phi(X))$ via $i^{*} \phi^{*}$. A straightforward check shows that $h$ is a homotopy of $\phi^{\prime} i$ and the identity.

In the rest of the section we fix an arrangement $\mathscr{A}$ and a sheaf $\mathscr{F}$ of $S$ modules on $L^{\mathrm{op}}$. Recall that $L_{0}=L^{\mathrm{op}} \backslash\{U\}$. There is a natural embedding $\mathscr{F}(U) \subset \Gamma\left(L_{0}, \mathscr{F}\right)=H^{0}\left(L_{0}, \mathscr{F}\right)$. This allows us to define

$$
\tilde{H}^{i}\left(L_{0}, \mathscr{F}\right)= \begin{cases}H^{i}\left(L_{0}, \mathscr{F}\right) / \mathscr{F}(U), & \text { if } i=0 ; \\ H^{i}\left(L_{0}, \mathscr{F}\right), & \text { otherwise. }\end{cases}
$$

Also if $I \in \operatorname{Spec} S$ and $X \in L$ we put $X(I)=\bigcap_{H \in \mathscr{A}_{X}, \alpha_{H} \in I} H$. Note that $X(I) \in L, X \subset X(I)$, and $X(I) \subset Y(I)$ for all $X \subset Y$ from $L$.

Now we recall an important definition from [ST].

Definition 1.3. The sheaf $\mathscr{F}$ is called local if for every $I \in \operatorname{Spec} S$ and $X \in L$ the localization at $I$ of the structure homomorphism $\mathscr{F}(X) \rightarrow \mathscr{F}(X(I))$ is an isomorphism.

Theorem 1.4. If $\mathscr{F}$ is local then for every $i \geq 0$ we have

$$
\operatorname{dim}_{S} \tilde{H}^{i}\left(L_{0}, \mathscr{F}\right) \leq \operatorname{dim} U .
$$

In particular if $\mathscr{A}$ is essential, i.e., $\operatorname{dim} U=0$, then $\tilde{H}^{i}\left(L_{0}, \mathscr{F}\right)$ is finite dimensional over $K$.

Proof. Fix an ideal $I \in \operatorname{Spec} S$ and put $L_{I}=\left\{X(I) \mid X \in L_{0}\right\}$. Since $\mathscr{F}$ is local the map $\phi: L_{0} \rightarrow L_{I}$ given by $X \mapsto X(I)$ and the sheaf $\mathscr{F}_{I}$ satisfy the conditions of Lemma 1.2. If $h t(I)<l-\operatorname{dim} U$ then $U(I) \neq U$, i.e., $U(I) \in L_{0}$. Also $(U(I))(I)=U(I)$ and hence $U(I)$ is the unique minimal element of $L_{I}$. Applying Lemmas 1.1 and 1.2 we obtain in this case that

$$
H^{i}\left(L_{0}, \mathscr{F}\right)_{I}=H^{i}\left(L_{0}, \mathscr{F}_{I}\right)=H^{i}\left(L_{I},\left.\mathscr{F}_{I}\right|_{L_{I}}\right)=0
$$

for every $i>0$. Also

$$
H^{0}\left(L_{0}, \mathscr{F}\right)_{I}=\Gamma\left(L_{0}, \mathscr{F}_{I}\right)=\Gamma\left(L_{I}, \mathscr{F}_{I}\right)=\mathscr{F}_{I}(U(I))=\mathscr{F}_{I}(U)
$$

whence

$$
\tilde{H}^{0}\left(L_{0}, \mathscr{F}\right)_{I}=\Gamma\left(L_{0}, \mathscr{F}_{I}\right) / \mathscr{F}_{I}(U)=0 .
$$

This implies that for every $i$ the height of an ideal from the support of $\tilde{H}^{i}\left(L_{0}, \mathscr{F}\right)$ is at least $l-\operatorname{dim} U$ and thus the annihilator of $\tilde{H}^{i}\left(L_{0}, \mathscr{F}\right)$ has the height at least $l-\operatorname{dim} U$. The result follows immediately.

To show that the above theorem is in fact a generalization of [ST, Proposition 6.10] we put $P(M ; x)=\sum_{k \geq 0} \operatorname{dim} M_{k} x^{k}$ (the Poincaré series of $M$ ) for a graded $S$-module $M$. Then we put $\chi(\mathscr{F})=\sum_{i \geq 0}(-1)^{i} P\left(\tilde{H}^{i}\left(L_{0}, \mathscr{F}\right) ; x\right)$ and call $\chi(\mathscr{F})$ the Euler characteristic of $\mathscr{F}$. 
Proposition 1.5. $\chi(\mathscr{F})=-\sum_{X \in L} \mu(X, U) P(\mathscr{F}(X) ; x)$.

Proof. Put $u=\operatorname{dim} U$. Following Baclawski [B] we apply the spectral sequence corresponding to the rank filtration of $L_{0}$ by the sets $L^{k}=\left\{X \in L_{0} \mid \operatorname{dim} X<\right.$ $k+u\}$. This is a fourth quadrant spectral sequence with

$$
E_{1}^{p, q}= \begin{cases}\bigoplus_{\operatorname{dim} X=p+u} \tilde{H}^{p+q-1}((U, X), \mathscr{F}(X)), & \text { if } p>1 ; \\ \bigoplus_{\operatorname{dim} X=u+1} \mathscr{F}(X), & \text { if } p=1, q=-1,\end{cases}
$$

where $(U, X)$ is an open interval of $L^{\text {op }}$, i.e., as a set $(U, X)=\{Y \in L \mid X<$ $Y<U\}$. Note that any closed interval of $L^{\text {op }}$ is a lattice whose opposite is a geometric lattice. Since the cohomology of a poset (with constant coefficients) coincides with the cohomology of the opposite poset (e.g., see [F, Theorem 3.1]) we can substitute $(X, U)$ instead of $(U, X)$ in the above formula where $(X, U)$ is viewed as an interval in $L$. Applying now [F, Theorem 4.1] we obtain the same result as in [B, Corollary 4.3]: the modules $\tilde{H}^{i}\left(L_{0} \mathscr{F}\right)$ are the homology modules of the complex

$$
0 \rightarrow C^{-1}=\mathscr{F}(U) \rightarrow C^{0} \rightarrow C^{1} \rightarrow \cdots \rightarrow C^{l-u-1} \rightarrow 0,
$$

where

$$
C^{i-1}=\bigoplus_{\operatorname{dim} X=i+u} \mathscr{F}(X)^{\oplus(-1)^{i} \mu(X, U)}
$$

for every $i=1, \ldots, l-u$. The result follows immediately.

Corollary 1.6 [ST, Proposition 6.10]. $(x-1)^{\operatorname{dim} U} \sum_{X \in L} \mu(X, U) P(\mathscr{F}(X) ; x)$ is a polynomial.

Proof. Theorem 1.4 and a well-known property of the dimension of graded modules imply that $(x-1)^{\operatorname{dim} U} P\left(\tilde{H}^{i}\left(L_{0}, \mathscr{F}\right)\right)$ is a polynomial for every $i$. Now the result follows from Proposition 1.5.

\section{COHOMOLOGY AND DEPTH OF $D$}

In this section we apply the general results of $\S 1$ to the sheaf $\mathscr{D}$. Note that $\mathscr{D}$ is local by [ST, Proposition 6.6]. We are primarily concerned with the projective dimension $\mathrm{pd}_{S}(D)$. By the Auslander-Buchsbaum theorem [M, p. 113] depth $D+\operatorname{pd}_{S}(D)=l$ where the depth of $D$ is defined with respect to the maximal homogeneous ideal of $S$. It turns out that the cohomology of $L_{0}$ with coefficients in $\mathscr{D}$ carries some information about $\mathrm{pd}_{S}(D)$ and thus about the depth of $D$. To explore this we first need the following as yet unpublished result of Terao [T4].

Lemma 2.1. $\operatorname{pd}_{S}(D(X)) \leq \mathrm{pd}_{S}(D)$ for every $X \in L$.

Proof. Fix an $X \in L$. Extending field $K$ to an infinite field if needed fix a point $p \in X$ in general position, i.e., satisfying the condition that if $p \in H$ for some $H \in \mathscr{A}$ then $X \subset H$. Let $\mathscr{M}$ be the maximal homogeneous ideal of $S$ and $\mathscr{P}$ the maximal ideal of $S$ corresponding to $p$. It follows from the graded 
version of Nakayama's Lemma that if the localization of a graded $S$-module $M$ is free over $S_{\mathscr{M}}$ then $M$ is free. This implies that $\operatorname{pd}_{S}(M)=\operatorname{pd}_{S_{\mathscr{L}}}\left(M_{\mathscr{M}}\right)$ which is true in particular for the graded $S$-module $D(X)$. By the choice of $p$ we have $U(\mathscr{P})=\bigcap_{p \in H} H=\bigcap_{X \subset H} H=X$. Thus since $\mathscr{D}$ is local we have $D_{\mathscr{P}}=D(U)_{\mathscr{P}} \simeq D(X)_{\mathscr{P}}$ whence

$$
\operatorname{pd}_{S_{\mathscr{P}}}\left(D(X)_{\mathscr{P}}\right)=\operatorname{pd}_{S_{\mathscr{P}}}\left(D_{\mathscr{P}}\right) \leq \operatorname{pd}_{S}(D) .
$$

Now it suffices to prove that

$$
\operatorname{pd}_{S_{\mathscr{P}}}\left(D(X)_{\mathscr{P}}\right)=\operatorname{pd}_{S_{\mathscr{M}}}\left(D(X)_{\mathscr{M}}\right) .
$$

For that we write $D(X)=\left(D(X)^{\prime} \otimes S_{X}\right) \oplus S^{k}$ where $D(X)^{\prime}=D\left(\mathscr{A}_{X}^{\prime}\right), \mathscr{A}_{X}^{\prime}$ is the arrangement induced by $\mathscr{A}_{X}$ in $V / X, S_{X}$ is the symmetric algebra of $X^{*}$, and $k=\operatorname{dim} X$ (e.g., see [ST, Proposition 5.8]). Let $T$ and $T_{X}$ be the automorphisms of the algebras $S$ and $S_{X}$ respectively generated by the translation of $X$ sending $p$ to 0 . Then $T(\mathscr{M})=\mathscr{P}$ whence $T$ induces a ring isomorphism $T_{1}: S_{\mathscr{M}} \rightarrow S_{\mathscr{P}}$. Now we define a group automorphism $T_{2}: D(X) \rightarrow D(X)$ by the identity on $D(X)^{\prime}$, by $T_{X}$ on $S_{X}$, and by $T^{k}$ on $S^{k}$. It is clear that $T_{2}$ is a $T$-isomorphism of modules, i.e., $T_{2}(s x)=T(s) T_{2}(x)$ for $s \in S$ and $x \in D(X)$. Thus $T_{2} \otimes T_{1}: D(X)_{\mathscr{M}} \rightarrow D(X)_{\mathscr{P}}$ is a $T_{1}$-isomorphism and (3) follows.

For the rest of the paper we assume that $\mathscr{A}$ is essential. It simplifies the results although they can be easily generalized to arbitrary arrangements.

Theorem 2.2. $\tilde{H}^{i}\left(L_{0}, \mathscr{D}\right)=0$ for every $i<l-1-\operatorname{pd}_{S}(D)$.

Proof. We again view $H^{i}=\tilde{H}^{i}\left(L_{0}, \mathscr{D}\right)$ as the homology groups of the complex $(1,2)$ for $u=0$. Denote by $B^{i}$ and $Z^{i}$ the submodules of $C^{i}$ of coboundaries and cocyles respectively. Now put $d=\operatorname{depth} D=l-\operatorname{pd}_{S}(D)$ and suppose that for some $i<d-1$ we have $H^{i} \neq 0$. Without loss of generality we can assume that $i$ is the least integer with this property, i.e., the sequence

$$
0 \rightarrow C^{-1} \rightarrow C^{o} \rightarrow \cdots \rightarrow B^{i} \rightarrow 0
$$

is exact. The short exact sequence $0 \rightarrow B^{i} \rightarrow Z^{i} \rightarrow H^{i} \rightarrow 0$ yields the long exact sequence

$$
\cdots \rightarrow \operatorname{Hom}\left(K, Z^{i}\right) \rightarrow \operatorname{Hom}\left(K, H^{i}\right) \rightarrow \operatorname{Ext}^{1}\left(K, B^{i}\right) \rightarrow \cdots
$$

where $K$ is viewed as an $S$-module via the projection $S \rightarrow S / \mathscr{M}=K$. Since $Z^{i}$ and $B^{i}$ are submodules of a module of positive depth their depths are positive too whence $\operatorname{Hom}\left(K, Z^{i}\right)=0$. Since $\mathscr{A}$ is essential depth $H^{i}=0$ by Theorem 1.5, i.e., $\operatorname{Hom}\left(K, H^{i}\right) \neq 0$. Thus $\operatorname{Ext}^{1}\left(K, B^{i}\right) \neq 0$, i.e., depth $B^{i}=$ 1. In particular since $B^{0}=D$ and depth $D \geq 2$ (e.g., see [T1]) we have $i>0$ and $Z^{0}=B^{0}=D$. Now applying the functor $\operatorname{Hom}(K,-)$ to each exact sequence $0 \rightarrow B^{j} \rightarrow C^{j} \rightarrow B^{j+1} \rightarrow 0$ for $j=i-1, i-2, \ldots, 0$ and 
using that depth $C^{j} \geq d$ by (2) and Lemma 2.1 , one concludes by induction that depth $Z^{j}=i+1-j$ (cf. [Y1], Lemma 4.2). Since $D=Z^{0}$ one gets depth $D=i+1<d$ which is a contradiction.

Corollary 2.3. If $\mathscr{A}$ is free then $\tilde{H}^{i}\left(L_{0}, \mathscr{D}\right)=0$ for every $i$ except maybe $i=l-1$. Thus

$$
\chi(\mathscr{D})=(-1)^{l-1} P\left(H^{l-1}\left(L_{0}, \mathscr{D}\right) ; x\right) .
$$

Example 2.4. Let $\mathscr{A}$ be a Boolean $l$-arrangement, i.e., an arrangement of all coordinate hyperplanes in some coordinate system. Then each $\mathscr{A}_{X}(X \in L)$ is the direct product of a Boolean $(l-k)$-arrangement $(k=\operatorname{dim} X)$ and the empty arrangement in $X$. Thus $P(D(X) ; x)=((l-k) x+k) /(1-x)^{l}$. Using that $L$ is a geometric lattice and thus $\mu(X, U)=(-1)^{k}$ we easily compute $\chi(\mathscr{D})$ from Proposition 1.5:

$\chi(\mathscr{D})=\sum_{X \in L}(-1)^{\operatorname{dim} X} \frac{(l-\operatorname{dim} X) x+\operatorname{dim} X}{(1-x)^{l}}=\sum_{i=0}^{l}(-1)^{i} \frac{((l-i) x+i)}{(1-x)^{l}}\left(\begin{array}{l}l \\ i\end{array}\right)=0$.

Since every Boolean arrangement is free we obtain from Corollary 2.3 that $\tilde{H}^{i}\left(L_{0}, \mathscr{D}\right)=0$ for every $i$. As an example of a non-Boolean free arrangement with 0 cohomology one can take the arrangement $\mathscr{A}_{1}$ from [O, Example 2.33].

In particular these examples show that $\tilde{H}^{\text {depth } D-1}\left(L_{0}, \mathscr{D}\right)$ can be 0 . However in certain cases this is impossible as the following result shows.

Theorem 2.5. Suppose that for all $X \in L \backslash\{U\}$ we have $\operatorname{pd}_{S}(D(X))<\operatorname{pd}_{S}(D)$. Then $H^{d-1}\left(L_{0}, \mathscr{D}\right) \neq 0$ where again $d=\operatorname{depth} D=l-\operatorname{pd}_{S}(D)$.

Proof. We use the notation from the proof of Theorem 2.2. Assume that $H^{d-1}=0$, i.e., the sequence $0 \rightarrow C^{-1} \rightarrow C^{0} \rightarrow \cdots \rightarrow B^{d} \rightarrow 0$ is exact. Consider for eac': $i=0,1, \ldots, d-1$ the short exact sequence $0 \rightarrow B^{i} \rightarrow$ $C^{i} \rightarrow B^{i+1} \rightarrow 0$. Using the functor $\operatorname{Hom}(K,-)$ as in Theorem 2.2 or [N, Theorem 20, p. 163] one can easily prove by induction on $i$ that $\operatorname{depth} B^{i}=d-i$. Note that the condition of the theorem implies that depth $C^{i}>d$ which is crucial for the proof. In particular for $i=d$ we obtain depth $B^{d}=0$ whence depth $C^{d}=0$ which is impossible.

Corollary 2.6. (i) If $\mathscr{A}$ is not free but $\mathscr{A}_{X}$ is free for every $X \in L \backslash\{U\}$ (e.g., if $\mathscr{A}$ is not free and $l=3$ ) then $H^{l-1}\left(L_{0}, \mathscr{D}\right) \neq 0$. (ii) If depth $D=2$ (e.g., if $\mathscr{A}$ is generic, see [RT] or [Y2]) then $H^{1}\left(L_{0}, \mathscr{D}\right) \neq 0$.

Proof. The first statement follows from Theorem 2.5 immediately. To prove the second statement it suffices to show that depth $D(X) \geq 2+\operatorname{dim} X$ for every $X \in L$. For that we represent $D(X)$ as in the proof of Theorem 2.2: $D(X) \simeq$ $D^{\prime}(X) \otimes S_{1} \oplus S^{k} \quad(k=\operatorname{dim} X)$. Since $D^{\prime}(X)=D\left(\mathscr{A}_{X}^{\prime}\right)$ for an arrangement $\mathscr{A}_{X}^{\prime}$ in $V / X$ we have $\operatorname{pd}_{S_{2}}\left(D^{\prime}(X)\right) \leq l-k-2$, where $S_{2}$ is the symmetric algebra of $(V / X)^{*}$. This implies that $\operatorname{pd}_{S}(D(X)) \leq l-k-2$ whence depth $D(X) \geq k+2$. 
Remark 2.7. One notices an obvious similarity between Theorems 2.2 and 2.5 and the characterization of the depth of a module in terms of local cohomology of the spectrum of a ring [Gr]. To explore this let us put $\chi=\operatorname{Spec} S$ and define $f: \chi \rightarrow L^{\text {op }}$ by $f(\mathscr{P})=\bigcap_{H \in \mathscr{A}, \alpha_{H} \in \mathscr{P}} H$. Since every set of $\alpha_{H}$ generates a prime ideal $f$ is surjective. Furthermore if $L^{X}=\{Y \in L \mid X \subset Y\}$ then $f^{-1}\left(L^{X}\right)=\left\{\mathscr{P} \in \chi \mid \alpha_{H} \notin \mathscr{P}\right.$ for every $H$ such that $\left.X \not \subset H\right\}$ which is an open affine subset of $\chi$. In particular $f$ is continuous and $f^{-1}\left(L_{0}\right)=\chi \backslash\{\mathscr{M}\}$. Now denote by $\tilde{D}$ the sheaf on $\chi$ generated by $D$. Since the sets $L^{X}$ form a basis of the topology on $L_{0}$ and since $f^{-1}\left(L^{X}\right)$ are affine one can prove that for every $i \geq 0$,

$$
H^{i}(\chi \backslash\{\mathscr{M}\}, \tilde{D}) \simeq H^{i}\left(L_{0}, f_{*} \tilde{D}\right)
$$

(cf. [H, Ex. III.4.1]). Together with [Gr, Proposition 2.2 and Theorem 3.8] (4) yields the equivalence of the following conditions:

(i) depth $D>d$,

(ii) $\tilde{H}^{i}\left(L_{0}, f_{*} \tilde{D}\right)=0$ for $i<d$.

Note that since $\mathscr{D}$ is a local sheaf we have $f_{*} \tilde{D} \simeq \mathscr{D} \otimes_{S} f_{*} \mathscr{O}$ where $\mathscr{O}$ is the structure sheaf on $\chi$. In particular $f_{*} \tilde{D}(X) \simeq D(X)_{\mathscr{P}(X)}$ where $\mathscr{P}(X)$ is the ideal of $S$ generated by all $\alpha_{H}$ for $H \in \mathscr{A} \backslash \mathscr{A}_{X}$. However no direct relation between $H^{i}\left(L_{0}, f_{*} \tilde{D}\right)$ and $H^{i}\left(L_{0}, \mathscr{D}\right)$ is known to me.

Remark 2.8. The proofs of Theorems 2.2 and 2.5 are valid for any local sheaf $\mathscr{F}$ of $S$-modules on $L^{\mathrm{op}}$ having the property that $\operatorname{pd}_{S}(\mathscr{F}(X)) \leq \operatorname{pd}_{S}(\mathscr{F}(U)) \leq$ $l-2$ for every $X \in L^{\text {op }}$. One should just substitute $\mathscr{F}(U)$ for $D$. In particular these theorems are true for the sheaves $\mathscr{D}^{p}(p=0,1, \ldots, l)$ generated by the modules $D^{p}$ defined in [ST].

\section{LATTICES OF FREE ARRANGEMENTS}

In this section we discuss certain conditions imposed on the intersection lattice $L$ of an arrangement $\mathscr{A}$ by the fact that $\mathscr{A}$ is free. The most famous condition of the type follows from Terao's theorem [T2]: the Poincare polynomial $\pi(L)$ of $L$ should be

$$
\pi(L ; t)=(1+t) \prod_{i=1}^{l-1}\left(1+b_{i} t\right)
$$

where $1, b_{1}, \ldots, b_{l-1}$ are the degrees of homogeneous generators of the free $S$-module $D$. Note that for a free arrangement $\mathscr{A}$ the Pioncare series of $D$ can be written as

$$
P(D ; x)=(1-x)^{-l}\left(x+\sum_{i=1}^{l-1} x^{b_{i}}\right)
$$

and thus is defined by $L$. 
Corollary 2.3 gives another condition on $L$. To formualte it let us say that a polynomial $p \in \mathbb{Z}[x]$ is nonnegative (and write $p \geq 0$ ) if all its coefficents are nonnegative.

Proposition 3.1. If an l-arrangement $\mathscr{A}$ is essential and free then $\chi(D)$ is a polynomial defined by $L$ and

$$
(-1)^{l-1} \chi(\mathscr{D}) \geq 0
$$

Proof. Theorem 1.4 implies that $\chi(\mathscr{D})$ is a polynomial. Since $\mathscr{A}$ is free all the arrangements $\mathscr{A}_{X}(X \in L)$ are free (see [T2]). Applying now (6) to the formula in Proposition 1.5 we see that $\chi(\mathscr{D})$ is defined by $L$. Besides Corollary 2.3 says that $(-1)^{l-1} \chi(\mathscr{D})$ is the Poincaré series of the $S$-module $H^{l-1}\left(L_{0}, \mathscr{D}\right)$ which completes the proof.

Corollary 3.2. If $\mathscr{A}$ is essential and free then

$$
(-1)^{l} \sum_{X \in L} \mu(X, U) \operatorname{dim} X \geq 0
$$

Proof. It suffices to notice that $\chi_{0}(\mathscr{D})=-\sum_{X \in L} \mu(X, U) \operatorname{dim} X$ is the 0-degree term of the polynomial $\chi(\mathscr{D})$ since $\operatorname{dim} D(X)_{0}=\operatorname{dim} X$ for every $X \in L$. Then the result follows from Propositions 1.5 and 3.1.

The criteria (7) and (8) allow us to prove that many geometric lattices cannot afford free arrangements although their Poincare series are of the form (5).

Example 3.3. Let $\mathscr{A}$ be a 3-arrangement of $k$ pencils of planes consisting of $r_{1}, r_{2}, \ldots, r_{k}$ planes respectively. Suppose that these pencils are in general position to each other. We will compute $\chi_{0}(\mathscr{D})$ for $\mathscr{A}$.

Put $s=\sum_{i \leq j \leq k} r_{i} r_{j}$. Since the pencils are in general position the planes of $\mathscr{A}$ intersect at $s+k$ distinct lines and each plane from the $i$ th pencil has exactly $1+\sum_{j \neq i} r_{j}$ of these lines in it. Thus

$$
\begin{aligned}
& \sum_{\operatorname{dim} X=1} \mu(X, U)=-s-k, \\
& \sum_{\operatorname{dim} H=2} \mu(H, U)=\sum_{i} r_{i} \sum_{j \neq i} r_{j}=2 s, \\
& \mu(V, U)=-1+s+k-2 s=-s+k-1,
\end{aligned}
$$

and finally

$$
\chi_{0}(\mathscr{D})=-s-k+4 s-3 s+3 k-3=2 k-3 .
$$

Applying (8) we conclude that for $k \geq 2$ the lattice of $\mathscr{A}$ cannot afford a free arrangement.

Note that among these lattices there are many families of those whose Poincaré polynomials factor as in (5). For example, if $k=3, r_{1}$ and $r_{2}$ are arbitrary and $r_{3}=r_{1} r_{2}+2\left(r_{1}+r_{2}\right)$ then it is easy to check that $\pi(L ; t)=$ $(1+t)\left(1+\left(r_{1}+r_{2}\right) t\right)\left(1+\left(r_{3}-2\right) t\right)$.

The case where $k=2, r_{1}=2$, and $r_{2}=5$ was first considered by $R$. Stanley in $[\mathrm{S}]$ as an example of a geometric nonsupersolvable lattice whose 
Poincaré polynomial factors. Later G. Ziegler proved in [Z, Example 3.7] that this arrangement is not free using his method of supersolvable resolutions. It is possible that his method could be applied to our more general class of lattices. We remark though that in general the considered lattices are not projectively unique and that for most of them a supersolvable resolution depends on an arrangement.

Remark 3.4. It is not necessary to know the factorization of $\pi(L)$ in order to check inequality (8) for a lattice $L$. However if the factorization is already known then it is sometimes easier to check other consequences of (7) first. In particular for $l=3$ let us write $\chi(\mathscr{D})$ explicitly:

$$
\begin{aligned}
\chi(\mathscr{D})=(x-1)^{-3}\left(x+x^{b_{1}}\right. & +x^{b_{2}}-\sum_{\operatorname{dim} X=1}\left(1+x+x^{m_{x}-1}\right) \\
& \left.+(2+x) \sum_{H \in \mathscr{A}}\left(m_{H}-1\right)+3 \mu(V, U)\right),
\end{aligned}
$$

where $m_{X}$ and $m_{H}$ are the number of planes passing through the line $X$ and the number of lines in the plane $H$ respectively. Then (9) and (7) imply

$$
\max \left\{b_{1}, b_{2}\right\} \geq \max _{\operatorname{dim} X=1}\left\{m_{X}-1\right\}
$$

which does not hold for all the cases in Example 3.3 where a factorization is given. It is not hard (using Example 3.3 for $k=3$ as a base) to construct examples where condition (8) holds but condition (10) does not. It would be interesting to find examples where (10) holds and (8) does not.

One can obtain other explicit inequalities from (7) if one represents $\chi(\mathscr{D})$ as a polynomial of $x-1$. For example, for $l=3$

$$
\chi(\mathscr{D})=\sum_{i \geq 0} c_{i}(x-1)^{i} \quad \text { with } c_{i}=\left(\begin{array}{c}
b_{1} \\
i+3
\end{array}\right)+\left(\begin{array}{c}
b_{2} \\
i+3
\end{array}\right)-\sum_{\operatorname{dim} X=1}\left(\begin{array}{c}
m_{X}-1 \\
i+3
\end{array}\right) .
$$

Let us remark also that it is easy to obtain condition (10) directly from the fact that for every $X \in L$ of dimension 1 there always exists a derivation $\theta_{X} \in D$ of degree $n-m_{X}$ linearly independent over $S$ of Euler derivation. Indeed one can put $\theta_{X}=\left(\prod_{H \in \mathscr{A} \backslash \mathscr{A}_{X}} \alpha_{H}\right) a_{X}$ for some $a_{X} \in X \backslash\{0\}$ (cf. [Y2]). This implies that $\min \left\{b_{1}, b_{2}\right\} \leq n-m_{X}$ for every $X$ which together with $b_{1}+b_{2}=n-1$ implies (10).

\section{REFERENCES}

[B] K. Baclawski, Whitney numbers of geometric lattices, Adv. in Math. 16 (1975), 125-138.

[F] J. Folkman, The homology group of a lattice, J. Math. Mech. 15 (1966), 631-636.

[Go] R. Godement, Topologie algébrique et théorie des faisceaux, Hermann, Paris, 1958.

[Gr] A. Grothendieck, Local cohomology, Lecture Notes in Math., vol. 41, 1967.

[H] R. Hartshorne, Algebraic geometry, Springer-Verlag, New York, 1977.

[M] H. Matsumura, Commutative algebra, Benjamin, New York, 1970. 
[N] D. Northcott, Finite free resolutions, Cambridge Univ. Press, London, 1976.

[O] P. Orlik, Introduction to arrangements, Amer. Math. Soc., Providence, R. I., 1989.

[RT] L. Rose and H. Terao, A free resolution of the module of logarithmic forms of a generic arrangement, J. Algebra (to appear).

[ST] L. Solomon and H. Terao, A formula for the characteristic polynomial of an arrangement, Adv. Math. 64 (1987), 305-325.

[S] R. Stanley, Supersolvable lattices, Algebra Universalis 2 (1972), 197-217.

[T1] H. Terao, Arrangements of hyperplanes and their freeness. I, J. Fac. Sci. Univ. Tokyo, Sect. IA Math. (2) 27 (1980), 293-312.

[T2] _ Generalized exponents of a free arrangements of hyperplanes and Shephard-ToddBrieskorn formula, Invent. Math. 63 (1981), 159-179.

[T3] _ Free arrangements of hyperplanes over an arbitrary field, Proc. Japan Acad. Ser. A Math. Sci. 59 (1987), 301-304.

[T4] _ On the homological dimensions of arrangements, in preparation.

[Y1] S. Yuzvinsky, Cohen-Macaulay seminormalizations of unions of linear subspaces, J. Algebra 132 (1990), 431-445.

[Y2] _ $A$ free resolution of the module of derivations for generic arrangements, J. Algebra (to appear).

[Z] G. Ziegler, Matroid representations and free arrangements, Trans. Amer. Math. Soc. (to appear).

Department of Mathematics, University of Oregon, Eugene, ORegon 97403 